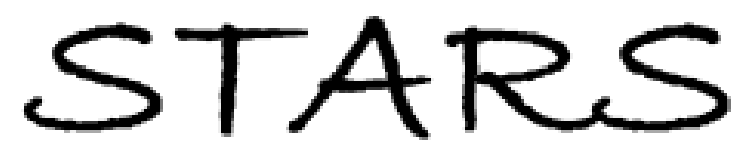

University of Central Florida

STARS

$1-1-2005$

\title{
Brownian dynamics simulation study of self-assembly of amphiphiles with large hydrophilic heads
}

Geuorgui K. Bourov

University of Central Florida

Aniket Bhattacharya

University of Central Florida

Find similar works at: https://stars.library.ucf.edu/facultybib2000

University of Central Florida Libraries http://library.ucf.edu

This Article is brought to you for free and open access by the Faculty Bibliography at STARS. It has been accepted for inclusion in Faculty Bibliography 2000 s by an authorized administrator of STARS. For more information, please contact STARS@ucf.edu.

\section{Recommended Citation}

Bourov, Geuorgui K. and Bhattacharya, Aniket, "Brownian dynamics simulation study of self-assembly of amphiphiles with large hydrophilic heads" (2005). Faculty Bibliography 2000 s. 4640.

https://stars.library.ucf.edu/facultybib2000/4640

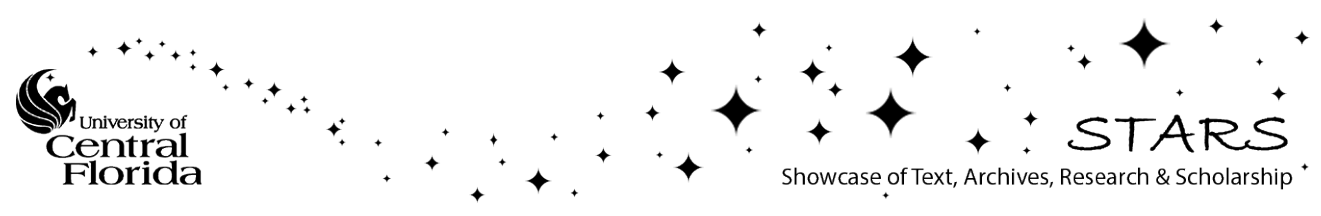




\section{Brownian dynamics simulation study of self-assembly of amphiphiles with large hydrophilic heads}

Cite as: J. Chem. Phys. 122, 044702 (2005); https://doi.org/10.1063/1.1834495

Submitted: 26 August 2004. Accepted: 26 October 2004 . Published Online: 04 January 2005

Geuorgui K. Bourov, and Aniket Bhattacharya

\section{ARTICLES YOU MAY BE INTERESTED IN}

The role of geometric constraints in amphiphilic self-assembly: A Brownian dynamics study

The Journal of Chemical Physics 119, 9219 (2003); https://doi.org/10.1063/1.1614210

Dynamics of entangled linear polymer melts: A molecular-dynamics simulation

The Journal of Chemical Physics 92, 5057 (1990); https://doi.org/10.1063/1.458541

Stochastic dynamics simulation of surfactant self-assembly

The Journal of Chemical Physics 106, 9850 (1997); https://doi.org/10.1063/1.473873

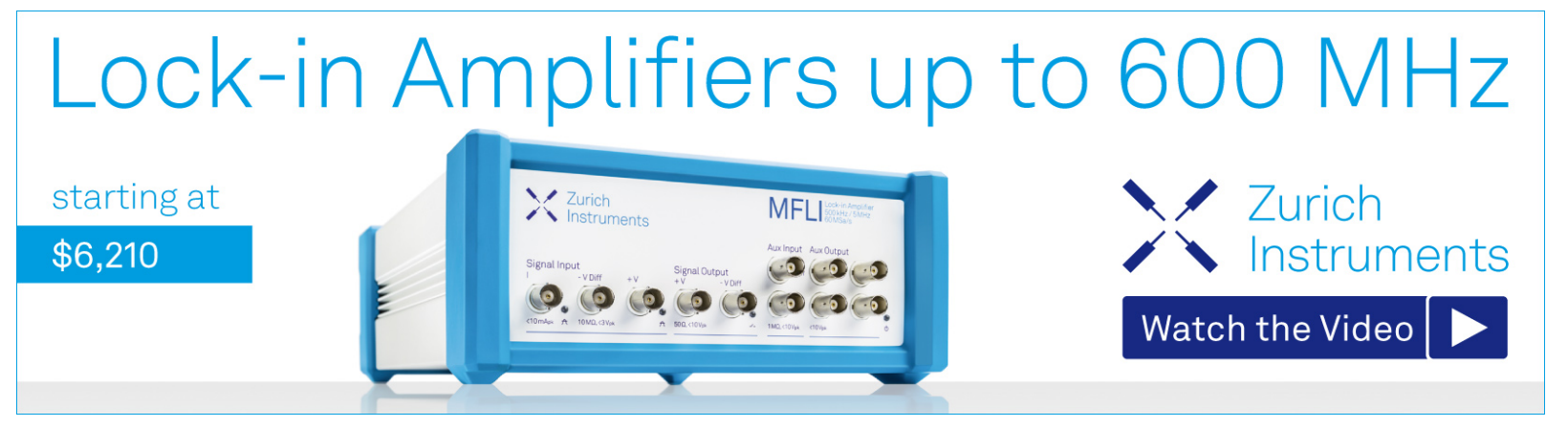

J. Chem. Phys. 122, 044702 (2005); https://doi.org/10.1063/1.1834495 


\title{
Brownian dynamics simulation study of self-assembly of amphiphiles with large hydrophilic heads
}

\author{
Geuorgui K. Bourov and Aniket Bhattacharya ${ }^{a)}$ \\ Department of Physics, University of Central Florida, Orlando, Florida 32816-2385
}

(Received 26 August 2004; accepted 26 October 2004; published online 4 January 2005)

\begin{abstract}
We have studied the effect of shape of an amphiphilic molecule on micellization properties by carrying out stochastic molecular dynamics simulation on a bead-spring model of amphiphiles for several sizes of hydrophilic head group with a fixed hydrophobic tail length. Our studies show that the effect of geometry of an amphiphile on shape and cluster distribution of micelles is significant. We find the critical micelle concentration increases with the increasing size of the hydrophilic head. We demonstrate that the onset of micellization is accompanied by (i) a peak in the specific heat as found earlier in the simulation studies of lattice models, and (ii) a peak in the characteristic relaxation time of the cluster autocorrelation function. Amphiphiles with larger hydrophilic head form smaller micelles with sharper cluster distribution. Our studies are relevant to the controlled synthesis of nanostructures of desired shapes and sizes using self-assembling properties of amphiphiles. (C) 2005 American Institute of Physics. [DOI: 10.1063/1.1834495]
\end{abstract}

\section{INTRODUCTION}

Self-assembling properties of amphiphilic molecules ${ }^{1-5}$ are recently being used as a synthesis platform in many nanotechnology related areas, e.g., energy and biological sensors, nanophotonics, and nanoelectronics. An amphiphilic molecule contains a hydrophilic and a hydrophobic moiety connected by chemical bonds. When dissolved in water, instead of a complete phase separation, they form spherical and cylindrical micelles, vesicles, bilayers, bicontinuous, and other structures. This self-assembling property of amphiphiles into different shapes can be suitably used to design templates for synthesis of devices at the nanometer length scale. ${ }^{6}$ The central idea is to develop bottom-up technology so that, starting from a given amphiphile, patterns over many length scales are realized.

A great variety of theoretical and numerical techniques have been developed to study amphiphilic self-assembly. Pioneering theoretical studies by Tanford ${ }^{1}$ and Israelachvili ${ }^{2}$ using basic principles of thermodynamics and statistical mechanics provide the energetics of micellization. On the other hand, simulation results with explicit incorporation of hydrophilic and hydrophobic elements of amphiphilic molecules have been able to provide a detail picture of certain shapes and dynamics of aggregation. A substantial amount of informations have been obtained through Monte Carlo (MC) simulations of lattice models. ${ }^{7-18}$ Compared to off-lattice models, lattice models are few orders of magnitudes faster on computer and simpler to implement. Recently, anomalous temperature dependence of critical micelle concentration (CMC) due to hydrogen bonding has been predicted using a lattice gas model. ${ }^{19}$ The lattice grand canonical Monte Carlo method has been able to provide phase diagram of different surfactants. ${ }^{20,21}$ It has been shown that by altering the ratio of

\footnotetext{
a) Author to whom correspondence should be addressed. Electronic mail: aniket@physics.ucf.edu
}

the hydrophilic and hydrophobic segments the surfactant system could be driven to a complete phase separation or micellization. ${ }^{21}$ The dependence of CMC on the hydrophobic and hydrophilic segments have been investigated in detail by the single-chain mean-field theory. ${ }^{22}$ Simulations in continuum using MC (Refs. 23 and 24) and molecular dynamics (MD) (Refs. 6 and 25-32) have provided great insights about amphiphilic self-assembly.

The specific geometry of an amphiphile affects the final structures in a nontrivial manner. It is only in the continuum models one can tune the hydrophilic and hydrophobic segments in any desired fashion and study self-assembly as a function of the shape and the size of the hydrophobic and hydrophilic building blocks. Off-lattice calculations are mostly done either with a bead-spring ${ }^{25,26}$ or a bead-stick model $^{31}$ where the successive beads are connected by either a spring or a rigid bond. Explicit incorporation of solvent particles usually is not done due to excessive computational cost; keeping the solvent particles severely restricts the system size and number of molecules for simulation. It is also generally accepted that the most of the equilibrium properties are captured well in the models with an implicit, continuum solvent.

The size of the head and tail beads are the same in most of the continuum studies using bead-spring and bead-stick models; only the number of the hydrophilic and hydrophobic beads are allowed to vary. Recently we have reported a two dimensional (2D) continuum stochastic molecular dynamic simulation study ${ }^{25,26}$ of micelle formation as a function of the hydrophilic head size. We observed that shapes and distributions of the micelles are profoundly altered as a function of the dimensionless packing parameter $\lambda=v / a_{0} l_{c}$, where $v$, $a_{0}$, and $l_{c}$ are the volume, the optimal head group area, and the critical chain length of an amphiphile, respectively. The preliminary $2 \mathrm{D}$ studies brought out interesting features. In this paper we extend calculations in three dimensions. These 
TABLE I. Interaction parameters for the amphiphiles.

\begin{tabular}{lccc}
\hline \hline Interaction & $r_{i j}^{c} / \sigma_{i j}$ & $\sigma_{i j}$ & $\epsilon_{i j}$ \\
\hline Head-head & $2^{1 / 6}$ & $\sigma_{t t}, 1.5 \sigma_{t t}, 1.58 \sigma_{t t}, 2 \sigma_{t t}$ & 1.0 \\
Head-tail & $2^{1 / 6}$ & $\frac{\sigma_{h h}+\sigma_{t t}}{2}$ & 1.0 \\
Tail-tail & 2.5 & 1.0 & 1.0 \\
\hline
\end{tabular}

studies enable us to compare our predictions with experiments, theoretical predictions, and simulation results obtained using other methods.

In the following section we provide the details of the simulation. In Sec. III we present our results. Section IV contains a summary and relevance of the present work and prospective related future work in this area.

\section{MODEL AND METHOD}

The details of the model and the method are given in reference. ${ }^{25,26}$ Here we briefly mention the information pertinent to the 3D simulations. An amphiphile is represented as $h_{m} t_{n}$ with $m$ hydrophilic heads $h$ and $n$ hydrophobic tails $t$ beads connected by $m+n-1$ bonds. We use Grest-Kremer type model $^{33}$ so that the nonbonded potential acting between any two beads are chosen to be Lennard-Jones (LJ) interactions and the interaction between successive beads is given by finite-extendable nonlinear elastic potential as given below:

$$
\begin{gathered}
U_{\mathrm{LJ}}^{i j}\left(r_{i j}\right)=4 \epsilon_{i j}\left[\left(\frac{\sigma_{i j}}{r_{i j}}\right)^{12}-\left(\frac{\sigma_{i j}}{r_{i j}}\right)^{6}-\left(\frac{\sigma_{i j}}{r_{i j}^{c}}\right)^{12}+\left(\frac{\sigma_{i j}}{r_{i j}^{c}}\right)^{6}\right], \\
r \leqslant r_{i j}^{c}, \\
U_{\text {chain }}\left(r_{i j}\right)=-\frac{1}{2} k R_{i j}^{2} \ln \left[1-\left(\frac{r_{i j}}{R_{i j}}\right)^{2}\right],
\end{gathered}
$$

where $r_{i j}^{c}$ is the cutoff distance beyond which the LJ interaction is set to be zero, $r_{i j}=\left|\vec{r}_{i}-\vec{r}_{j}\right|$ and $\vec{r}_{i}, \vec{r}_{j}$ are the locations of the $i$ th and $j$ th monomers respectively. Amphiphilicity in this model is introduced by a repulsive cutoff distance for the head-head and head-tail $\left(r_{h h}^{c}=2^{1 / 6} \sigma_{h h}, r_{h t}^{c}=2^{1 / 6} \sigma_{h t}\right)$, and an attractive cutoff for the tail-tail interaction $\left(r_{t t}^{c}=2.5 \sigma_{t t}\right) . k$ and $R_{i j}$ are the force constant and the length parameters of the potential. We have chosen $k=30\left(\epsilon_{t t} / \sigma_{t t}^{2}\right), R_{i j}=1.5 \sigma_{i j}$, and $\epsilon_{i j}=1$. The choice of the LJ parameters are summarized in Table I. Each monomer is coupled to a heat bath and its equation of motion is

$$
m_{i} \ddot{r}_{i}=-\vec{\nabla} U_{i}-\Gamma \vec{r}_{i}+\vec{W}_{i}(t),
$$

where $U_{i}=\sum_{i \neq j}\left[U_{\mathrm{LJ}}^{i j}\left(r_{i j}\right)+\sum_{j=i \pm 1} U_{\text {chain }}\left(r_{i j}\right)\right], m_{i}$ is the mass of the $i$ th particle, $\Gamma$ is the monomer friction coefficient, and $\vec{W}_{i}(t)$ describes the random force of the heat bath acting on each monomer as a Gaussian white noise with zero mean satisfying the fluctuation-dissipation relation $\left\langle\vec{W}_{i}(t)\right.$ $\left.\cdot \vec{W}_{j}\left(t^{\prime}\right)\right\rangle=6 m_{i} k_{B} T \Gamma \delta_{i j} \delta\left(t-t^{\prime}\right)$. The stationary solutions of above equations of motion produce Boltzmann distribution and therefore the simulated system has canonical ensemble thermodynamical constrains. Additionally, we use reduced

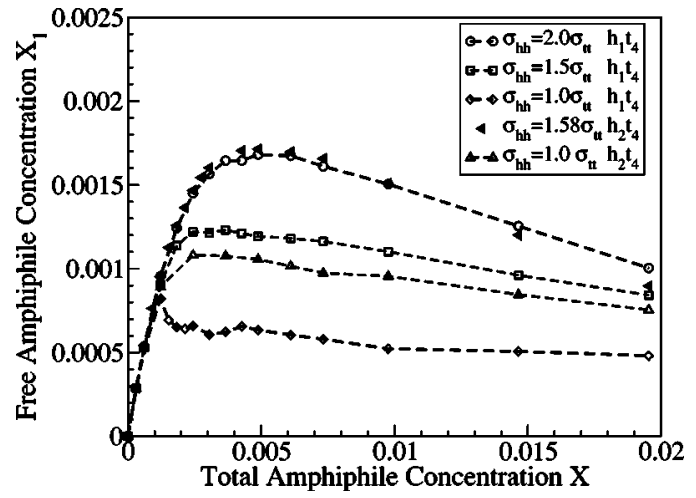

FIG. 1. The concentration of free amphiphiles $X_{1}$ as a function of the total concentration of amphiphiles $X$ for different head sizes. The symbols $\bigcirc, \square$, and $\diamond$ correspond to $\sigma_{h h}=2 \sigma_{t t}, \sigma_{h h}=1.5 \sigma_{t t}$, and $\sigma_{h h}=\sigma_{t t}$ for $h_{1} t_{4}$. The symbols $\triangle$ and $\triangle$ correspond to $h_{2} t_{4}$ with $\sigma_{h h}=1.58 \sigma_{t t}$ and $\sigma_{h h}=1.0 \sigma_{t t}$, respectively. The lines drawn through the points serve as a guide to the eye.

units throughout this study; the unit of length is $\sigma_{t t}$, the unit of time is $\tau=\sigma_{t t}\left(m / \epsilon_{t t}\right)^{1 / 2}$, and the unit of temperature is $\epsilon_{t t} / k_{B}$ where $k_{B}$ is the Boltzmann constant. All beads have equal mass which is set to unity. The parameter $\Gamma=1.0$, and the integration time step $\Delta t=0.01 \tau$. We have kept the reduced temperature at $k_{B} T / \epsilon=0.9$ for all the results reported here.

\section{RESULTS}

The simulations are carried out in a $32 \times 32 \times 32$ box with periodic boundary conditions. Typical length of the runs are $(5-10) \times 10^{6} \mathrm{MD}$ steps excluding $10^{6}$ equilibrating $\mathrm{MD}$ steps. The maximum number of the chains in the box is 1600. We have considered two types of amphiphiles $h_{1} t_{4}$ and $h_{2} t_{4}$. Amphiphiles of different shapes are simulated by choosing $\sigma_{h h}=\sigma_{t t}, \sigma_{h h}=1.5 \sigma_{t t}, \sigma_{h h}=1.58 \sigma_{t t}$, and $\sigma_{h h}$ $=2 \sigma_{t t}$, respectively (see Table I). We have used a link-cell list and a fast Gaussian random number generator to expedite the calculations.

\section{A. Critical micelle concentration}

First we study how the number density $X_{1}$ of single amphiphiles (unimers) varies with the total concentration $X$ of the amphiphiles for different head sizes. The results are shown in Fig. 1. $X_{1}$ saturates with an increase of the total amphiphilic concentration $X$. The value of $X$ around the knee of the graph defines the CMC beyond which energy contribution dominates over entropic effects and in principle clusters of all sizes can form. Consistent with our previous 2D studies, ${ }^{25,26}$ we find that the CMC increases with larger size of the hydrophilic head. This behavior has been observed experimentally $^{34}$ and in lattice Monte Carlo simulation by Guerin and Szleifer ${ }^{22}$ and Rodriguez-Guadarrama et al. ${ }^{16}$

Next we compare amphiphilic molecules of different shapes but of same fixed volume. We approximate the volume of an amphiphile as $\sum_{i=1, l} 4 / 3 \pi \sigma_{i i}^{3}$. We consider amphiphilic molecule $h_{2} t_{4}$ with different $\sigma_{h h}$ and compare the results with those obtained for $h_{1} t_{4}$ for the following two cases. First we study the amphiphile $h_{2} t_{4}$ with $\sigma_{h h}$ $=1.58 \sigma_{t t}$. The volume of this molecule is the same as that 


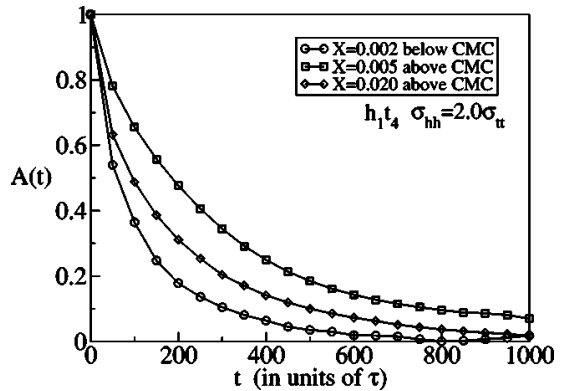

FIG. 2. Time dependence of $A(t)$ for different concentrations $X$ for $h_{1} t_{4}$.

of $h_{1} t_{4}$ with $\sigma_{h h}=2.0 \sigma_{t t}$. The dependence of $X_{1}$ on $X$ is shown in Fig. 1. This is almost indistinguishable for the corresponding curve for $h_{1} t_{4}$ with $\sigma_{h h}=2.0 \sigma_{t t}$. The second case that we consider is $h_{2} t_{4}$ with $\sigma_{h h}=1.0 \sigma_{t t}$. This molecule and the amphiphile $h_{1} t_{4}$ with $\sigma_{h h}=1.26 \sigma_{t t}$ have the same volume. It is seen from Fig. 1 that the CMC for $h_{2} t_{4}$ with $\sigma_{h h}=\sigma_{t t}$ falls in between those for $h_{1} t_{4}$ with $\sigma_{h h}$ $=\sigma_{t t}$ and $\sigma_{h h}=1.5 \sigma_{t t}$. From these two examples we may conclude that the steric volumes of hydrophilic and hydrophobic segments of an amphiphile roughly controls its CMC for a given temperature.

\section{B. Autocorrelation function $A(t)$}

The free amphiphilic molecules and the molecules in aggregates are in dynamic equilibrium. To characterize the dynamics of aggregation we monitor the size of the aggregate $N(t)$ where a chain resides at time $t$ and calculate the tracer autocorrelation function ${ }^{35} A(t)$ defined as

$$
A(t)=\frac{\left\langle N\left(t_{0}+t\right) N\left(t_{0}\right)\right\rangle-\left\langle N\left(t_{0}\right)\right\rangle^{2}}{\left\langle N^{2}\left(t_{0}\right)\right\rangle-\left\langle N\left(t_{0}\right)\right\rangle^{2}} .
$$

The averages $\langle\cdots\rangle$ are taken over all the chains and for the all time of the simulation. A useful parameter is the characteristic time $\tau_{c}$ defined as the time needed for $A(t)$ to reach value of $e^{-1}$. First, we show the dependence of $A(t)$ on total concentration $X$ for $h_{1} t_{4}$ with $\sigma_{h h}=2 \sigma_{t t}$ and recover the usual result; namely, below CMC, $A(t)$ increases with concentration and above CMC relaxation is faster as shown in Fig. 2. The dependence of $A(t)$ on the head size reveals important characteristics of micellization as shown in Fig. 3. The characteristic time $\tau_{c}$ decreases dramatically for larger head group. It shows that the hydrophilic tails inside the core are

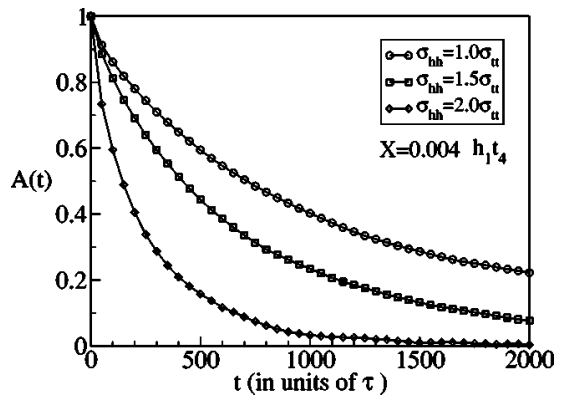

FIG. 3. Behavior of $A(t)$ at $X=0.004$ for three different geometries $\sigma_{h h}$ $=\sigma_{t t}(\bigcirc), \sigma_{h h}=1.5 \sigma_{t t}(\square)$, and $\sigma_{h h}=2 \sigma_{t t}(\diamond)$ for $h_{1} t_{4}$.

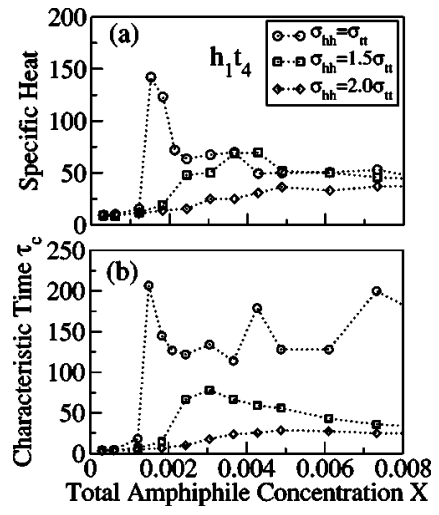

FIG. 4. Specific heat (a) and characteristic time $\tau_{c}$ (b) as a function of amphiphilic concentration for $h_{1} t_{4}$ type molecules. The lines serve as a guide.

more loosely bound for amphiphiles with large heads. We find that there is a strong correlation between the characteristic autocorrelation time $\tau_{c}$ and the onset of micellization. Energy fluctuations in a $N-V-T$ ensemble are proportional to the specific heat of the system $C_{v}=k_{\mathrm{B}} T^{2}\left\langle\Delta E^{2}\right\rangle$. Previously one of us observed in a lattice model that the $C_{v}$ exhibits a peak at the onset of micellization. ${ }^{14}$ Similar behavior has been noticed by Shida and Henriques. ${ }^{15}$ Figure 4 displays the variation of the specific heat with the concentration. The peak of the graph for each case occurs at the CMC (Fig. 1) for the given system. On the same figure [Fig. 4(b)] we show the behavior of characteristic time $\tau_{c}$ as a function of the total concentration $X$. We notice that $\tau_{c}$ also exhibits a peak at the CMC. The time dependence of $\tau_{c}$ on total amphiphilic concentration is consistent with Fig. 2. Hence in an experiment one can measure CMC by obtaining the cluster autocorrelation time using a tagged amphiphile. Calculation of $\tau_{c}$ has another practical and useful application; namely, calculating the correct errors in correlated data set. During our simulations we have calculated the standard deviation $\sigma$ of a quantity $m$ using the following expression:

$$
\sigma=\sqrt{\frac{2 \tau_{c}}{t_{\max }}\left(\overline{m^{2}}-\bar{m}^{2}\right)}
$$

where $\tau_{c}$ is the above defined autocorrelation time, $\bar{m}$ represent averaged value of $m$, and $t_{\max }$ is the total time of the simulation.

\section{Cluster shapes and distribution}

During the simulation we have monitored the normalized probability distribution $p_{n}$ of micelles as a function of the cluster size $n$ given by

$$
p_{n}=\frac{\left(\frac{X_{n}}{n}\right)}{\sum_{i} \frac{X_{i}}{i}},
$$

$\sum_{i} p_{i}=1$ 


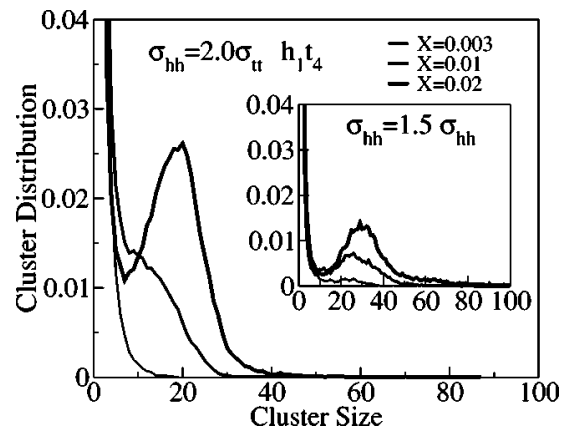

FIG. 5. Cluster distribution as a function of the cluster size for two different head group sizes $\sigma_{h h}=2 \sigma_{t t}, \sigma_{h h}=1.5 \sigma_{t t}$ (inset), and for different concentrations.

where $X_{n}$ is the total concentration of amphiphiles for clusters of size $n$. We also calculate the eigenvalues $\lambda_{i}$ of the moment of inertia tensor $I_{\alpha \beta}$ given by

$$
I_{\alpha \beta}=\sum_{i=1}^{n} m_{i}\left(X_{\mathrm{CM}}^{\alpha}-x_{i}^{\alpha}\right)\left(X_{\mathrm{CM}}^{\beta}-x_{i}^{\beta}\right),
$$

which provides informations about the shapes of the micelles. Here $X_{\mathrm{CM}}^{\alpha}$ and $x_{i}^{\alpha}$ are the $\alpha$ th coordinates of the center of mass of the cluster and the $i$ th particle of the cluster, respectively. $\alpha, \beta$ can take values 1,2 , and 3 along the three different axes. Diagonalization of the inertia tensor yields three principal moments of inertia $\lambda_{i}$. We use $L_{i}=\sqrt{\lambda_{i}}, i$ $=1,2$, and 3 as the definition of characteristic lengths. During the simulation we first sort three principal moment of inertia in the descending order of their magnitudes and $\left(L_{1}\right.$ is the largest and $L_{3}$ the smallest). The ratios $\left(L_{1} / L_{2}\right)$ and $\left(L_{1} / L_{3}\right)$ reveal information for the shapes of the micelle. For a perfect spherical micelle these two ratios will be unity.

The probability distributions for the cluster sizes as a function of different concentration (greater than the CMC) are shown in Fig. 5 for $\sigma_{h h}=2.0 \sigma_{t t}$ and $\sigma_{h h}=1.5 \sigma_{t t}$ (inset). A peak in the cluster distribution is seen as expected. The new feature of our studies is the dependence of the peak on the size of the hydrophilic head group. At $X=0.02$ for $\sigma_{h h}$ $=2.0 \sigma_{t t}$ the peak occurs around $n \sim 20$, whereas, for $\sigma_{h h}$ $=1.5 \sigma_{t t}$ the peak shifts toward $n \sim 30$. It is also noticeable that the distribution for $\sigma_{h h}=2.0 \sigma_{t t}$ is much sharper $\left(p_{n}\right.$ $=0.025$ at $n=20)$ compared to the corresponding distribution for $\sigma_{h h}=1.5 \sigma_{t t}\left(p_{n}=0.0125\right.$ at $\left.n=30\right)$. This is our second new result.

Table II shows the details of the characteristic lengths for the most probable aggregates (for the size which corresponds to the peak of each cluster size distribution) for two different head geometries $\left(\sigma_{h h}=1.5 \sigma_{t t}\right.$ and $\left.\sigma_{h h}=2.0 \sigma_{t t}\right)$, respectively. The ratios of the characteristic lengths are shown in Fig. 6. We notice $L_{1} / L_{2} \simeq 1$ for all aggregate sizes. This implies that the almost perfect circular shape across these

TABLE II. Characteristic lengths for most probable cluster distributions.

\begin{tabular}{lcccccc}
\hline \hline$\sigma_{h h}$ & Cluster size & $L_{1}$ & $L_{2}$ & $L_{3}$ & $\left(L_{1}+L_{2}\right) / 2$ & $\left(L_{1}+L_{2}\right) / 2 L_{3}$ \\
\hline $2.0 \sigma_{t t}$ & 20 & 6.47 & 6.16 & 2.79 & 6.315 & 2.26 \\
$1.5 \sigma_{t t}$ & 30 & 5.22 & 4.95 & 2.68 & 5.09 & 1.90 \\
\hline \hline
\end{tabular}

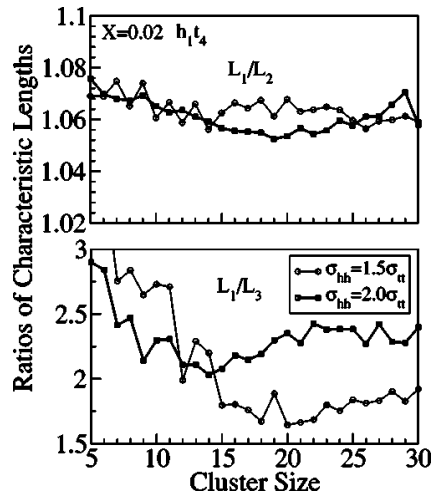

FIG. 6. Variation of the shape parameters $L_{1} / L_{2}$ (top) and $L_{1} / L_{3}$ (bottom) as a function of the cluster size for two different head sizes $\sigma_{h h}=1.5 \sigma_{t t}$ and $\sigma_{h h}=2 \sigma_{t t}$. The lines serve as a guide.

two axes. We also notice that the ratio $\left(L_{1}+L_{2}\right) / 2 L_{3}$, is slightly larger for $\sigma_{h h}=2 \sigma_{t t}$. These values suggest that the most probable micelles in both systems are ellipsoidal but the micelles made off amphiphiles with smaller head size $\left(\sigma_{h h}=1.5 \sigma_{t t}\right.$ are slightly more spherical. A snapshot of 3D simulation box is given in Fig. 7. Two typical aggregates from our simulations are isolated and shown in Fig. 8.

The cluster distributions and characteristic ratios for $h_{1} t_{4}$ with $\sigma_{h h}=2.0 \sigma_{t t}$ and $h_{2} t_{4}$ with $\sigma_{h h}=1.58 \sigma_{t t}$ are shown in Fig. 9 for comparison. These two types of amphiphilic molecules are chosen since they have the same volume of the hydrophilic segments. Earlier we found in Fig. 1 that their CMC values are close. Here we notice that the cluster distributions are almost indistinguishable. Additional information obtained from shape parameters reveal very similar properties as shown in Fig. 6 . The ratio $\left(L_{1}+L_{2}\right) / 2 L_{3}$, is slightly larger for $\sigma_{h h}=2.0 \sigma_{t t}$ around the peak of the cluster distribution. It will be interesting to find the optimal size of the hydrophilic head for which both the characteristic ratios are close to unity leading to formation of near spherical micelles.

\section{CONCLUSION}

In summary, we have studied the role of the head group geometry in amphiphilic self-assembly for a bead-spring model of flexible amphiphiles using Brownian dynamics

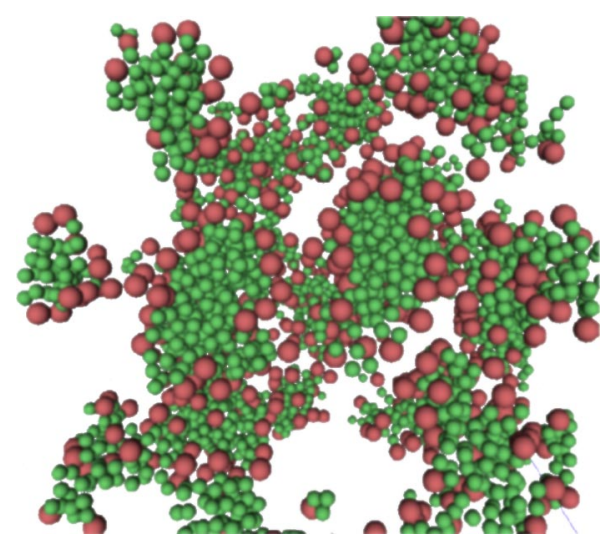

FIG. 7. A snapshot of 3D simulation box with periodic boundary conditions for $h_{1} t_{4}$ with $\sigma_{h h}=1.5 \sigma_{t t}$. 

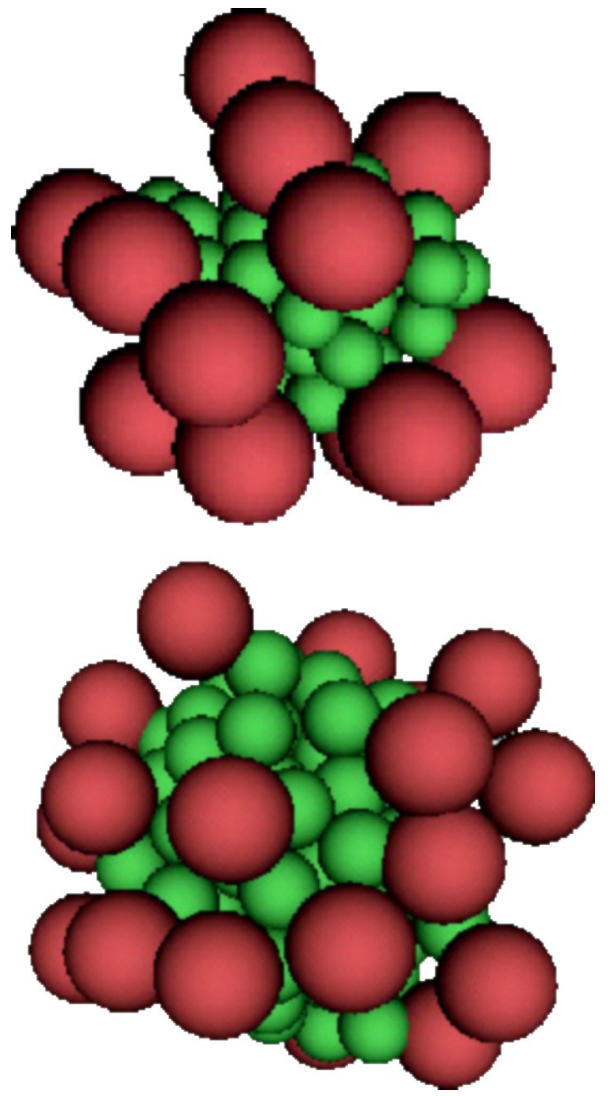

FIG. 8. Snapshots of micelles formed by $h_{1} t_{4}$ (a) for 12 chains with $\sigma_{h h}$ $=2.0 \sigma_{t t}$ (top) and (b) for 20 chains with $\sigma_{h h}=1.5 \sigma_{t t}$ (bottom).

simulation. The principal idea of this work is to demonstrate that the shape and size of the micelles can be controlled by varying the size of the hydrophilic head only. We show that the CMC increases for larger size of the hydrophilic head segment. This is consistent with the experimental findings. For a given length of the hydrophobic tail, micelles of desired shape can be obtained by a proper choice of the hydrophilic head. Amphiphiles with larger heads form micelles with very sharp cluster distribution. In addition, we find that a peak in the specific heat and in the characteristic autocorrelation time at the onset of micellization is a generic feature

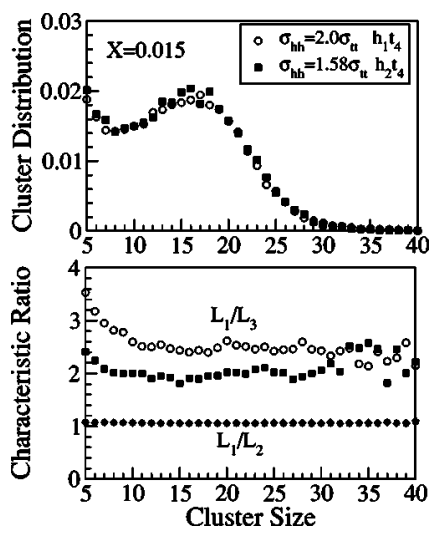

FIG. 9. Cluster distribution (top) and characteristic ratios (bottom) as a function of the cluster size for two different head group geometries $h_{1} t_{4}$ with $\sigma_{h h}=2.0 \sigma_{t t}$ and $h_{2} t_{4}$ with $\sigma_{h h}=1.58 \sigma_{t t}$ for $X=0.02$. of the self-assembly. Previous simulation studies by other groups based on lattice and off-lattice models discussed dependence of amphiphilic self-assembly of concentration, temperature, and chain length. In this paper we have focused on a systematic investigation of geometric effects in amphiphilic self-assembly. We find that the geometric effects are rather nontrivial and a simulation based knowledge can be very useful for nano mask fabrication and other surfactant mediated templating methods. We are extending these investigations for other types of amphiphiles, e.g., double tailed surfactant which are the building blocks of lipid bilayers. We have seen that for the same temperature and concentration studied here double tailed surfactants after initial formation of large micelles eventually form bilayers. ${ }^{36}$ Since vesicles and bilayers are the key ingredients of cell membranes, synthesis of these structures will have enormous applications. Our simulations are aimed to provide useful information for controlled synthesis of these structures by suitable choice of amphiphilic geometry.

\section{ACKNOWLEDGMENTS}

The research reported here was supported in part by a grant from the National Science Foundation NIRT (ENG/ ECS and CISE/EIA) under Grant No. 0103587. The authors thank Weili Luo and Kevin Belfield for various discussions.

${ }^{1}$ C. Tanford, The Hydrophobic Effect: Formation of Micelles and Biological Membranes (Wiley, New York, 1980).

${ }^{2}$ J. Israelachvili, Intermolecular and Surface Forces, 2nd ed. (Academic, London, 1992).

${ }^{3}$ S. A. Safran, Statistical Thermodynamics of Surfaces, Interfaces, and Membranes (Addison-Wesley, New York, 1994).

${ }^{4}$ Surfactants and Polymers in Aqueous Solution, edited by B. Jönsson, B. Lindman, K. Holmberg, and B. Kronberg (Wiley, New York, 1998). See Chaps. 2 and 4.

${ }^{5}$ P. C. Hiemenz and R. Rajagopalan, Principles of Colloid and Surface Chemistry (Dekker, New York, 1997).

${ }^{6}$ Z. Zhang, M. Horsch, M. Lamm, and S. Glotzer, Nano Lett. 3, 1341 (2003).

${ }^{7}$ R. G. Larson, L. E. Scriven, and H. T. Davis, J. Chem. Phys. 83, 2411 (1985); R. G. Larson, ibid. 89, 1642 (1988); 91, 2479 (1989); 96, 7904 (1992); J. Phys. II 6, 1441 (1996).

${ }^{8}$ C. M. Care, J. Phys. C 20, 689 (1987); J. Chem. Soc., Faraday Trans. 83, 2905 (1987); D. Brindle and C. M. Care, ibid. 88, 2163 (1994); J.-C. Desplat and C. M. Care, Mol. Phys. 87, 441 (1996).

${ }^{9}$ P. H. Nelson, G. C. Rutledge, and T. A. Hatton, J. Chem. Phys. 107, 10777 (1997).

${ }^{10}$ A. T. Bernardes, V. B. Henriques, and P. M. Bisch, J. Chem. Phys. 101, 645 (1994); J. Phys. II 6, 169 (1996).

${ }^{11}$ M. Kenward and M. D. Whitmore, J. Chem. Phys. 116, 3455 (2002).

${ }^{12}$ A. D. Mackie, A. Z. Panagiotopoulos, and I. Szleifer, Langmuir 13, 5022 (1997).

${ }^{13}$ A. Bhattacharya and S. D. Mahanti, J. Phys.: Condens. Matter 12, 6141 (2000).

${ }^{14}$ A. Bhattacharya and S. D. Mahanti, J. Phys.: Condens. Matter 13, L861 (2001).

${ }^{15}$ C. S. Shida and V. B. Henriques, J. Chem. Phys. 115, 8655 (2001).

${ }^{16}$ L. A. Rodriguez-Guadarrama, S. K. Talsania, K. K. Mohanty, and R. Rajagopalan, Langmuir 15, 437 (1999).

${ }^{17}$ V. Kapila, J. M. Harris, P. A. Deymier, and S. Raghavan, Langmuir 18, 3728 (2002).

${ }^{18}$ M. A. Floriano, E. Caponetti, and A. Z. Panagiotopoulos, Langmuir 15, 3143 (1998)

${ }^{19}$ H. Bock and K. E. Gubbins, Phys. Rev. Lett. 92, 135701 (2003).

${ }^{20}$ A. Z. Panagiotopoulos, M. A. Floriano, and S. K. Kumar, Langmuir 18, 2940 (2002). 
${ }^{21}$ S. Salaniwal, S. K. Kumar, and A. Z. Panagiotopoulos, Langmuir 19, 5164 (2003).

${ }^{22}$ C. B. E. Guerin and I. Szleifer, Langmuir 15, 7901 (1999).

${ }^{23}$ A. Milchev, A. Bhattacharya, and K. Binder, Macromolecules 34, 1881 (2001).

${ }^{24}$ A. Galindo, C. Vega, E. Sanz, L. G. McDowell, E. de Miguel, and F. J. Blas, J. Chem. Phys. 120, 3957 (2004).

${ }^{25}$ G. K. Bourov and A. Bhattacharya, J. Chem. Phys. 119, 9219 (2003).

${ }^{26}$ A. Bhattacharya and G. Bourov, Effect of Packing Parameter on Amphiphilic Self-Assembly: A Brownian Dynamics Study, Computer Simulation Studies in Condensed Matter Physics, Vol. 16, edited by D. P. Landau, S. P. Lewis, and H.-B. Schüttler (Springer, Heidelberg, 2004).

${ }^{27}$ D. R. Rector, F. van Swol, and J. R. Henderson, Mol. Phys. 82, 1009 (1994).

${ }^{28}$ B. Palmer and J. Liu, Langmuir 12, 746 (1996); 12, 6015 (1996); B.
Palmer, J. Liu, and J. Virden, ibid. 15, 7426 (1999).

${ }^{29}$ P. K. Maiti, Y. Lansac, M. A. Glaser, N. A. Clark, and Y. Rouault, Langmuir 18, 1908 (2002).

${ }^{30}$ J.-B. Maillet, V. Lachet, and P. V. Coveney, Phys. Chem. Chem. Phys. 1, 5277 (1999).

${ }^{31}$ F. K. von Gottberg, K. A. Smith, and T. A. Hatton, J. Chem. Phys. 106, 9850 (1997)

${ }^{32}$ S. Bogusz, R. M. Venable, and R. Pastor, J. Phys. Chem. 105, 8312 (2001)

${ }^{33}$ G. S. Grest and K. Kremer, Phys. Rev. A 33, 3628 (1986).

${ }^{34}$ L. Ambrosone, L. Costantino, G. D'Errico, and V. Vitagliano, J. Colloid Interface Sci. 190, 286 (1997).

${ }^{35}$ T. Haliloglu and W. L. Mattice, Chem. Eng. Sci. 49, 2851 (1993).

${ }^{36} \mathrm{G}$. K. Bourov and A. Bhattacharya (unpublished). 